\title{
Information Sheets and Informed Consent Forms for Clinical Study Participants: Towards Standardised Recommendations?
}

\author{
Olivier Chassany ${ }^{1}$, Micheline Bernard-Harlaut ${ }^{2}$, Gilles Guy $^{3}$, Nathalie Billon ${ }^{4}$ and the participants in Round \\ Table $N^{\circ} 3$, Giens XXIV ${ }^{\star}$
}

1 DRRC, PH-HP, Hôpital Saint Louis, Paris, France

2 CNAFAL, Conseil National Associations Familiales Laïques, Paris, France

3 Chemire Le Gaudin, France

4 Sanofi-Aventis, Paris, France

Text received 15 october 2008; accepted 20 november 2008

\section{Keywords:}

informed consent;

information;

clinical trials;

patients;

biomedical research; writing

\begin{abstract}
Subjects taking part in biomedical research must be provided with legible and intelligible information enabling them to freely give their informed consent. At present, sponsors tend to provide many different types of information, not all of which is directly connected with or indeed really informative about studies for those taking part.

As a result of this observation, a round table was convened during the Clinical Pharmacology meetings to deliberate on the creation of a charter concerning the drafting of information documents for biomedical research participants as well as a code of good practice for the preparation of such documents.

Recommendations were made based on the efforts of the various working groups concerned, such as the French National Conference of Ethics Committees (CNCP), users' representatives, patients associations and the French industrial and institutional sponsors association (CPI), together with proposals contained in the literature.

The deliberations of the round table may be subsumed under the following 3 categories and 14 points:

1) Format: design, drafting rules, layout, table of contents, glossary

2) Content: introductory page, description of the study, risks, benefits

3) Regulatory aspects: legal aspects, CNIL message (data protection), financial aspects, conflict of interests, model and varied signatures.

This document should help make research in France more attractive and it was decided after the Clinical Pharmacology meeting to submit the charter and related documents for approval by the various actors involved: DGS (Direction Générale de la Santé), Afssaps (Agence Française de Sécurité Sanitaire des Produits de Santé), CNCP, CPI and LEEM (Les entreprises du médicament).

Once the charter has been validated, it will be made available to Sponsors and Ethics Committees in order to ensure greater uniformity and legibility regarding information given to study subjects.
\end{abstract}

\section{Introduction}

There is now an urgent need to write these recommendations. Although persons taking part in biological research require legible and intelligible information in order to be able to give their free and informed consent, sponsors have a marked tendency to

\footnotetext{
* For a list of participants, see the end of the article.
}

provide many types of different information not always directly associated with or truly informative about the research subject.

Ethics committees are often confronted with poorly written documents submitted without any real reflection on ethical matters and in spite of this, they must ensure that a coherent position is adopted by experts and that the decisions made are consistent from one ethics committee to another. 
Recommendations for good practice concerning the creation of information sheets were summed up in 8 points for discussion by the round table. These recommendations are based on the efforts of different working groups such as those of the French National Conference of Ethics Committees (CNCP), ${ }^{[1]}$ users representatives, patients associations, industrial and institutional sponsors associations (CPI), and recommendations in the literature. ${ }^{[2-9]}$

Information sheets and consent forms are intended for persons wishing to take part in biomedical research and they must provide an understanding of the aims, benefits and risks, constraints and actual conduct of the project. These documents must be written concisely in French, using simple, unambiguous language. They must contain only useful information, without repetition, and they must distinguish clearly between health care and clinical research. Even if signed by study subjects, informed consent forms do not constitute legally binding contracts.

The text of these recommendations must signal true commitment on the part of sponsors and ethics committees alike. The principles are based on recognition of and respect for persons taking part in research, thereby indicating close adhesion to ethical principles on the part of sponsors and ethics committees in the service of clinical research. This document must enhance the attractiveness of clinical research in France, and the members of the round table agreed on the need to present the charter and documents following the clinical pharmacology meetings and to have them approved by all actors involved, namely the DGS (Direction Générale de la Santé), Afssaps (Agence française des sécurité sanitaire des produits de santé), CNCP, CPI and LEEM (Les entreprises du médicament).

\section{Methods}

The aims of the round table were to set down a charter concerning both the preparation of information documents for subjects taking part in biomedical research and good writing practice. This initial study concerns only general cases and deliberately leaves aside the specific questions raised by clinical trials involving children, adolescents and elderly subjects.

\subsection{Design}

The person responsible for drafting information documents must have extensive knowledge of the study and of French culture, and the best-equipped person is thus the study project manager. If the study protocol and annexes have initially been drafted in English, the study product manager must check the translation.
A reading committee must check the draft, and we feel it is essential that the principal investigator should review the information document.

The drafting of this document should take account of the following 5 key points.

\subsubsection{The distinction between Research and Care}

In a setting of care, the doctor is concerned solely with the interest of the patient as an individual; he is guided exclusively by the patient's direct interests. In a research situation, however, the approach is different since the aim is to produce scientific knowledge. In this case, attention is directed towards the group to which the subject belongs.

Recognition of this specific nature of biomedical research necessitates a distinction between the research situation and the care situation.

Thus, the terms "investigating doctor", "volunteer", "study product" or "eligibility" are used to describe a biomedical situation and the terms "doctor", "patient", "drug" or "treatment" are used for a care setting.

\subsubsection{All consent must be informed}

The universally accepted rule today concerning informed consent expresses the obligation on the part of the investigating doctor to inform the person being invited to take part in a study of the nature and content of the research project and of the aims. Thus, the initial information must first deal with the specific features of the research situation.

This requirement is particularly important given the frequency of research projects conducted in a health care setting, where there is an increased risk of confusion between care procedures and research procedures; for example, merely indicating that blood will be withdrawn does not indicate the type of act that will be carried out.

The first requirement concerning information is to allow the participant to understand that he or she is being invited to take part in a biomedical research project.

There is little point worrying how many details the patient remembers if the distinction is not clearly made between a study protocol and a health care protocol, or between the study product and a drug.

\subsubsection{The information must be relevant}

In this context, the key requirement of the information given is its ability to allow the potential participant to decide whether or not to take part in the study. 
Information must be intelligible if it is to be used as the basis for good decisions.

The idea, often repeated, that it is necessary to "explain everything" is false. Relevant information is that information which is useful for decision-making. All information likely to affect a person's decision must be considered essential (e.g. existence of risk, consequences on quality of life of participation in the study, constraints associated with the study conduct, etc.).

\subsubsection{The information must be intended specifically for the person being asked to take part in the study}

Recognition of the specificity of the situation concerning biomedical research and changes in legal measures assigning greater responsibility to sponsors suggest greater importance of "written" procedures. An information document can no longer be regarded as a simple accessory used for oral presentations with the responsibility falling on the investigating doctor. This document must express the sponsor's undertaking to perform high-quality research with complete respect for the subjects involved.

Subjects may judge the degree of consideration in which they are held by the quality of the documents given to them. They are the ones to whom the document is addressed, and this intention must be clear from the text.

\subsubsection{Written and oral information must be homogeneous}

Regulatory measures stipulate the conditions under which information is to be provided: oral information by the investigating doctor (or a doctor representing the latter) as well as written documents summarising the information given orally. The written and oral information must be homogeneous. Consistency between the written information and the oral information given ensures uniform presentation of the content. Problems of continuity can affect a subject's decision-making process due to the confusion it produces.

\subsection{Rules for writing}

a-Phrase length

Complete sentences must not contain more than 30 words; the average is 25 words.

\section{$b$-Construction of phrases}

Straightforward phrases should be preferred. Constructions involving successive subordinate clauses should be avoided. Presentation of a single piece of new information in each sentence results in short and easily understood sentences.

\section{c-Specialised vocabulary}

The amount of specialised vocabulary requiring an explanation depends on the medical knowledge of the person concerned by the medical speciality or disease. Some patients have a good knowledge of their disease, in which case it is somewhat clumsy to oversimplify. Where necessary, attempts to simplify specialised terms and/or notions should be based on existing tools directed at the general public.

$$
\text { d-Style }
$$

A neutral style should be adopted and the active tense should be preferred to the passive.

\section{e-Compliance with terminology}

Care should be taken to use terms and expressions included in a pre-set glossary.

This recommendation is particularly important regarding items that distinguish different situations (biomedical research versus care situations). Particular attention must be paid to the use of the following terms: treatment, drug, investigating doctor, biomedical research.

\section{$f$ - Layout and typesetting}

Proper care should be taken to ensure good application of the rules concerning capitalisation, punctuation, etc. Parentheses should be avoided and of course mistakes in spelling and grammar must be corrected.

A standard font of size 11/12 should be used and paragraphs should be clearly separated and spaced, with the sponsor's logo figuring discreetly on the page, if at all.

\subsection{Layout}

Layout is important to enable those invited to take part in the study so as to demonstrate the sponsor's interest and respect for them. The title page must give value to the document, and it should contain the study title and the sponsor's name.

\subsection{List of contents}

A list of contents must be included, particularly in large or long documents. For example, we propose the following table of contents:

1. Study name and title

2. Glossary

3. Introduction

4. Table of contents

5. General information on the study product

6. General information on the study

7. Visits

8. Risks 
9. Benefits

10. Medical alternatives

11. Interpretation of the study

12. Potential costs

13. Rights and obligations

14. Regulatory considerations

15. Summary table of visits

\subsection{Glossary}

It is also important to add a glossary for the information of the person invited to take part in the study (to help with the assimilation of any oral information given). This glossary may comprise:

Terms used in all biomedical research (consent, biomedical research, investigating doctor, sponsor, visits, information, inclusion) as well as their definition.

Terms specific to the biomedical research described in the document. Each term must be defined using resources intended for the "general public" (e.g. dictionaries or websites).

In the french version of this round table, the readers could find an annexe, in french language with examples for words to use or proscribe.

\section{Results}

\subsection{Introductory page}

This should be set out as a letter addressed to the person in courteous and polite terms, taking care to avoid such expressions as "Dear Sir" or "Dear Patient". We propose that the person be addressed simply as: Madame, Ms or Sir.

The study aim must clearly indicate how the subject being invited to take part is actually concerned by the study and it must indicate the time accorded for reflection, although inclusion of this information is not strictly necessary. Mention must be made of the subject's freedom to take part in or refuse to take part in the study as well as the undertaking by the study doctor to provide best possible care.

\subsection{Description of the study}

The description of the study must be addressed directly to the person (using the "you" form), and it must include details of the study methodology, clearly indicating how the study is to be performed using diagrams and timetables. Management procedures after the study or in the event of premature study discontinuation must be indicated. The same description rules must be applied to additional studies.
Mention must be made of the duration of participation for the participant as well as the total study duration. It may be helpful to include a patient card clearly indicating certain points in the study protocol.

We suggest that this part be structured as follows, with certain sections and sub-sections that may be used from one study to another, and others that must be adapted by the person responsible for drafting the information sheet.

\subsubsection{General information on the study product}

In accordance with the recommendations concerning the use of simple and unambiguous terms, the expression "study product" is used to describe the subject of the study in the case of drug trials. The expression "study treatment" should be avoided except where it refers to products already evaluated and in use.

\subsubsection{Type and status}

The type (molecule, device, technique) of the study product and its status as regards regulatory position and practice [whether or not Marketing Authorization (MA) has been granted, temporary use authorisation, whether already marketed, in which countries, in which indication(s)].

\subsubsection{Mechanism of action}

The mode and mechanism of action of the study product or device must be presented in clear and simple terms. Technical and scientific explanations should be used only in the oral information section for persons requiring such elucidation.

\subsubsection{What is already known about the study product}

Regardless of the stage of development, the results of previous studies must be indicated. Footnotes for instance may be used to refer the study participant to more precise details on the studies conducted. Where applicable, links should be provided allowing complete consultation of the studies cited.

\subsubsection{General information on the study}

This section focuses on information useful for presentation of the study, regardless of the personal aspects of participation in the study. This information should be organised as follows:

\subsubsection{Context of the study}

The general context and "health"-oriented nature of the study should be indicated. The reasons for undertaking such a study should be given (reasons and aims).

\subsubsection{Aims}

The general study aims must be indicated (what the study is primarily seeking to establish); this section should focus on the 
principal objective rather than multiplying the secondary scientific objectives. The study objectives should not be confounded with the ultimate study aims (the aim may be a safety study for a general study of which the ultimate purpose is to test a molecule with a view to producing a future drug).

\subsubsection{Methodological aspects}

The number of centres and countries must be stated, together with the number of persons concerned, the scheduled duration and study dates, and the general study design (e.g. double-blind, placebo-controlled, randomised). The terminology may be defined in the glossary.

\subsubsection{Visits}

The study conduct must be explained to the person in terms of visits, classified according to type and chronological order: selection visits/inclusion and study visits/end-of-study visits/follow-up visit(s).

\subsubsection{The selection visit}

This section should include a list of procedures to be carried out on study participants as stipulated in the study protocol and classified by type.

\subsubsection{Inclusion visit}

The role of this visit in the study conduct (confirmation of participation for example) must be indicated with details of all examinations, tests and work-ups scheduled.

\subsubsection{Subsequent visits}

This section should contain a description of the practical and significant aspects of the study from the point of view of the person taking part (quantity and type of examinations, constraints, disadvantages, monitoring, administration conditions, etc.). In order to shorten this section, reference may be made to a table of visits indicating all acts to be carried out, which should be inserted on the final page of the document.

\subsubsection{End-of-study visit}

All examinations, tests and work-ups scheduled for this visit must be indicated.

\subsubsection{Follow-up visit(s)}

The number and times of these visits must be specified, together with the examinations, tests and work-ups to be performed.

\subsubsection{Calendar of study visits}

A graphic representation of the study is preferable, adapted to the individual characteristics of the study (frequency and type of visits, content and details of examinations, presentation of different possibilities according to the study design, etc.) This graphic representation must be clear and intelligible and present the study conduct from the point of view of the person taking part.

\subsubsection{Termination of the study}

Participation in biomedical research is entirely voluntary and is not a legally binding initiative comparable to a formal or contractual agreement. Such participation may be discontinued for a number of reasons. This section must distinguish between several different reasons for termination of the study:

- at the initiative of the sponsor, the investigator or the health authorities,

- medical reasons,

- wish of the participant (withdrawal).

\subsection{Risks}

We suggest that you group together information concerning adverse effects, examinations and risks associated with pregnancy.

The "risk" section comprises 4 sub-sections:

\subsubsection{Unwanted effects and events}

This information must be presented clearly and honestly and must provide the participant with authentic information.

$>$ Molecules under development

Mention must be made of any adverse events (AE) already observed in prior studies. There is no point presenting the results of animal studies, in vitro or in vivo, except for molecules in phase I or II. In this case, the data presented must provide real, intelligible and useful information for the participant.

\section{$>$ Device}

Information must be provided about any risk of radiation and must not play down the importance of such issues.

\section{Products already marketed}

The package insert may be used but text taken from the summary of product characteristics is not suitable.

\section{Associated drugs not supplied}

The adverse effects of associated drugs not supplied (nonexperimental drugs) do not need to be presented since they are prescribed in accordance with the marketing authorisation and form part of the treatment administered normally as part of the routine health care given.

In general, the presentation will depend on the severity and frequency of the effects in question (thus for instance "aplasia" should not be innocently inserted between "nausea" and "fatigue"). 


\subsubsection{Risks associated with the study procedures}

These may be potential consequences associated with the medical examinations as well as the conditions under which they are performed, presented in terms of discomfort for participants (particularly as regards pain). The information concerning risks associated with the study examinations and procedures must faithfully reflect the real risk of the examinations and the gravity of the disease. For example, do not exaggerate the risks associated with obtaining blood samples, do not overlook the risks associated with anaesthesia if an examination required by the research project calls for such anaesthesia and if this would not have been given under normal care conditions.

\subsubsection{Risks associated with specific situations}

Details must be given of specific risks engendered by specific populations or situations (allergies, pregnancy, etc.). It is important to adapt the recommendations to specific study populations (young patients, terminally ill patients, etc.), thus information relating to pregnancy or breast feeding must be formulated in accordance with the study population, the potential risk of the drugs and the disease. Similarly, the contraceptive methods proposed must be realistic and suited to the study subject.

\subsubsection{Precautions to be taken}

This section should be completed with a practical presentation of the measures to be taken in the event of problems.

Thus an indication should be given of the procedures to be followed in the case of an $\mathrm{AE}$ or warning signs (e.g. aplasia/fever, bleeding/anticoagulant, etc.). Clear mention should be made of all directly observable signs.

\subsection{Benefits}

There are three types of benefits and these must be described as precisely as possible. The presentation of potential benefits (particularly personal therapeutic benefits) must not be used to pressurise subjects into participating.

\subsubsection{Personal therapeutic benefits}

Such benefits must be described in practical terms (e.g. "possible reduction of your risk of heart attack"). Further, the conditions under which such benefits may be observed must be explained, and thus personal therapeutic benefits may be observed if the study product is efficacious and the person has been included in the "study product" group. It must also be stated that such benefits do not exist for members of the placebo group.

\subsubsection{Personal non-therapeutic benefit}

Inclusion in the study results in "improved" management in relation to the standard care and treatment. This advantage must be discussed with regard to the study characteristics (type of examinations, diagnostic possibilities, etc.).

\subsubsection{Collective benefit}

In principle, there is always a collective benefit for the community from all types of biomedical research. (NB: Biomedical research is defined by law as improvement in biological and medical knowledge.)

\section{Legal aspects}

Legal aspects correspond to legal references in the French Public Health Code; it is therefore not useful to list the articles in question and their references. A standard phrase such as "We are inviting you to take part in a biomedical research project covered by the laws intended for your protection" is sufficient.

It will be necessary for a working group of legal specialists to be created in order to check whether the phrase commonly found regarding "transfer of data outside Europe" is sufficient to ensure the protection of participants. In particular, sponsors must be encouraged to add the following phrase: "The sponsor agrees to ensure a level of security in keeping with French or European law regarding the transmission of data concerning you to other countries".

\section{Information concerning CNIL (French Data Protection Agency)}

The CNIL wording cannot be changed, despite the fact that this text does not meet our recommendations concerning intelligibility and readability. It would be helpful to convene a working group of CNCP, DGS, LEEM and CNIL members to reflect on a more accessible text.

\section{Financial aspects}

Information concerning patents and intellectual property are not mandatory since they are not intended to protect the study participant.

\section{Insurance}

Regarding compensation, it is essential to indicate that an insurance policy has been taken out by the sponsor, although it is 
not necessary to indicate the policy number. There is no need to indicate measures or precautions intended to limit the extent of the insurance in the information sheet.

\section{Costs for participants}

Any costs (these must be clearly explained) shall be borne entirely by the sponsor. In order to uphold study subject confidentiality with respect to the sponsor, a third-party agent must be used in order to manage cost reimbursement.

\section{Payment}

Regardless of context, payment may be made to participants in consideration of constraints imposed by the study. In this case, an explanation must be provided in the information document.

Cover of the study subject by a general social security regime

The information document and informed consent form must insist on the need for cover by the social security system (eligibility).

\section{Conflict of interests}

The Declaration of Helsinki recommends that investigators declare any conflicts of interests to patients, although this may be done orally. It should be noted that any explanation to participants of the different sources of finance, particularly in public-private partnerships, is generally a source of confusion. Officially, conflicts of interest on the part of the investigator consist of any ties between the investigator and the sponsor studying the molecule or medical device, as well as ties with any industrial competitors.

Depending on these various elements, we recommend that rather than each investigator declaring a conflict of interest in writing, investigators may declare their interests orally.

\section{Multiple and varied signatures}

The document must be signed by the participant. The participant may have recourse to a witness only where he or she is unable to write. For minors or incapable majors, the signature of the legal representative alone is necessary. In emergency situations or for comatose patients, only the person authorised by the law will be allowed to sign.

Although the investigator's signature is not required by law, the round table thus recommends that it be added since it signals the investigator's commitment.

Each page of the information document must include the study references (date, version), although initialling of each indi- vidual page is not necessary. The information sheet and informed consent form may comprise a single indivisible document.

\section{Conclusion}

These recommendations should be a real source of aid for sponsors on drafting of information sheets and for ethics committees when analysing study projects submitted to them. They must be approved by all partners involved (Coordination of Institutional Sponsors (CPI), LEEM, CNCP and by the various authorities (DGS, AFSSAPS).

\section{Participants}

Bernard Alberola (Wyeth Pharmaceuticals), Jean-François Bergmann (Médecine Interne A, Hôpital Lariboisière, Paris), Micheline Bernard-Harlaut (CNAFAL), Christian Cailliot (Amgen), Hélène Chappuy (Hôpital Necker Enfants Malades, Paris), Olivier Chassany (DRRC, AP-HP, Hôpital Saint Louis, Paris), Xavier Coron (Sanofi-Aventis), Jean-Luc Cracowski (CIC, Inserm, CHU, Grenoble), Hervé Decousus (Médecine Interne et Thérapeutique, CHU Hôpital de Bellevue, Saint Etienne), Christophe Demonfaucon (AFTOC, Chateaufort), Catherine Fagard (Inserm, Université Bordeaux), Hugues Fischer (Association de patients, Paris), Odile Gelpi (Hospices civils, Lyons), Danielle Golinelli (Directions Générale de la Santé), Marie-Laurence Gourlay (Afssaps), Gilles Guy (Chemire le Gaudin), Yves Juillets (LEEM), Rémi Le Coent (GlaxoSmithKline), Christian Legrand (URAEUS, Fontainebleau), Monique Malchiodi (Laboratoires Roche), Anne-Laure Morin (Avocate, Paris), Véronique Nalet (Lilly France), Alain Olympie (La Maison des MICI, Paris), François Rivaille (laboratoires Servier), Claire Sibenaler (LLEM), Isabelle Thizon de Gaulle (Sanofi-Aventis).

\section{References}

1. Groupe de travail sur le document d'information et le consentement éclairé de la Conférence Nationale des Comités de Protection des Personnes. Synthèse des travaux.

2. Paris A, Cracowski JL, Ravanel N, et al. Lisibilité de l'information écrite destinée aux sujets se prêtant à une recherche biomédicale. Presse Med 2005; 34: 13-8

3. Paris A, Cracowski JL, Maison P, et al. Impact of French "Comités de Protection des Personnes" on the readability of informed consent documents (ICD) in biomedical research: more information, but not better information. Fundam Clin Pharmacol 2005; 19: 395-9

4. Sharp SM. Consent documents for oncology trials: does anybody read these things? Am J Clin Oncol 2004; 27: 570-5

5. Wendler D. Can we ensure that all research subjects give valid consent ? Arch Intern Med 2004; 164: 2201-4 
6. Flory J, Emanuel E. Interventions to improve research participants' understanding in informed consent for research: a systematic review. JAMA 2004; 292: 1593-601

7. Bjørn E, Rossel P, Holm S. Can the written information to research subjects be improved? An empirical study. J Med Ethics 1999; 25: 263-7

8. Dresden GM, Levitt MA. Modifying a standard industry clinical trial consent form improves patient information retention as part of the informed consent process. Acad Emerg Med 2001; 8: 246-52
9. Paris A, Nogueira da Gama Chaves D, Cornu C, et al. Improvement of the comprehension of written information given to healthy volunteers in biomedical research: a single-blind randomized controlled study. Fundam Clin Pharmacol 2007; 21: 207-14

Correspondence and offprints: Nathalie Billon, Sanofi Aventis, 9 boulevard Romain Rolland, 75159 Paris Cedex 14, France.

E-mail: nathalie.billon@sanofi-aventis.com 